\title{
Mapping of local argon impingement on a virtual surface: an insight for gas injection during FEBID
}

\author{
H. D. Wanzenboeck · G. Hochleitner • \\ J. Mika • M. M. Shawrav • M. Gavagnin • \\ E. Bertagnolli
}

Received: 26 May 2014/Accepted: 27 August 2014/Published online: 5 November 2014

(C) The Author(s) 2014. This article is published with open access at Springerlink.com

\begin{abstract}
During the last decades, focused electron beam induced deposition (FEBID) has become a successful approach for direct-write fabrication of nanodevices. Such a deposition technique relies on the precursor supply to the sample surface which is typically accomplished by a gas injection system using a tube-shaped injector nozzle. This precursor injection strategy implies a position-dependent concentration gradient on the surface, which affects the geometry and chemistry of the final nanodeposit. Although simulations already proposed the local distribution of nozzle-borne gas molecules impinging on the surface, this isolated step in the FEBID process has never been experimentally measured yet. This work experimentally investigates the local distribution of impinging gas molecules on the sample plane, isolating the direct impingement component from surface diffusion or precursor depletion by deposition. The experimental setup used in this work maps and quantifies the local impinging rate of argon gas over the sample plane. This setup simulates the identical conditions for a precursor molecule during FEBID. Argon gas was locally collected with a sniffer tube, which is directly connected to a residual gas analyzer for quantification. The measured distribution of impinging gas molecules showed a strong position dependence. Indeed, a $300-\mu \mathrm{m}$ shift of the deposition area to a position further away from the impingement center spot resulted in a $50 \%$ decrease in the
\end{abstract}

Electronic supplementary material The online version of this article (doi:10.1007/s00339-014-8755-y) contains supplementary material, which is available to authorized users.

H. D. Wanzenboeck $(\bowtie) \cdot$ G. Hochleitner · J. Mika ·

M. M. Shawrav · M. Gavagnin · E. Bertagnolli

Institute for Solid State Electronics, Vienna University of

Technology, Floragasse 7/1 - E362, 1040 Vienna, Austria

e-mail: Heinz.Wanzenboeck@tuwien.ac.at precursor impinging rate on the surface area. With the same parameters, the precursor distribution was also simulated by a Monte Carlo software by Friedli and Utke and showed a good correlation between the empirical and the simulated precursor distribution. The results hereby presented underline the importance of controlling the local precursor flux conditions in order to obtain reproducible and comparable deposition results in FEBID.

\section{Introduction}

As a maskless and resistless direct-write method, focused electron beam induced deposition (FEBID) has attracted increasing interest over the last decade [1,2], especially as it allows to directly deposit three-dimensional structures on arbitrary surfaces [3-5]. In spite of the versatility of this method, the progress of FEBID suffers from a lack in reproducibility among the results of individual researchers. This has two main reasons: (1) the high complexity of the deposition mechanism itself and (2) the different instrumentation used.

The high complexity of the entire mechanism is due to the electron-substrate interaction (finally leading to deposition) and the surface precursor dynamics [6, 7]. The precursor's surface coverage on a specific deposition location results from the dynamic equilibrium between addition and depletion of precursor, i.e., between increase and decrease of the local precursor coverage. During FEBID, the surface coverage can increase (1) by adsorption from the gas phase and (2) by incoming surface diffusion from neighboring locations. The surface coverage can decrease (1) by outgoing surface diffusion, (2) by desorption into gas phase, or (3) by intentional precursor 
consumption during the deposition reaction. In this work, we focus exclusively on the experimental investigation of the adsorption step isolated from the other process steps. In FEBID, the adsorption is mainly governed by the precursor supply via the nozzle of a gas injection system (GIS). As stated previously, the wide variety of different GIS instrumentation implicating different precursor supply conditions is the second source for the low comparability among FEBID results.

Most FEBID systems use microtube-based GISs [8] that lead to a gradient in the precursor distribution in dependence on the different distance of the surface position from the injection nozzle. The local precursor coverage on the deposition spot determines whether the process is conducted in the electron-limited regime or in the precursorlimited regime $[9,10]$. This way the precursor coverage determines the deposits minimum dimensions and geometry [11], the deposition rate [12, 13], and its chemical composition $[13,14]$. Not only for fundamental insights but also for practical reproducibility of each deposition one must know and reproduce the same precursor density at the electron exposed deposition areas. One of the difficulties in comparing FEBID results is the fact that the different precursor injection systems come with a different spatial distribution of the precursor. Unfortunately, it has become a common deficit not to the describe nozzle geometries and precursor injection conditions in publications. In this work we will experimentally demonstrate that-as already expected-dissimilar impinging geometries indeed have a massive impact on the local precursor coverage on the sample, so that a detailed GIS description is essential for meaningful interpretation of results.

To shine some light on this issue of precursor supply from the GIS, the precursor flux and the precursor distribution on the sample surface have been topic of several studies with particular focus on simulation. The geometry of the deposited material has been simulated assuming a homogenous localized gas pressure [10], supposing a Langmuir adsorption model [15] and even considering the contributions of surface diffusion [16, 17]. In addition, Rack et al. $[2,10]$ have implemented gas-handling algorithms on their 3D Monte Carlo based simulation of FEBID. However, unless simulations do not pay tribute to the change in local precursor coverage their ability to simulate the real deposition from any nozzle-based GIS can only remain limited.

Driven by the need for a deeper understanding on the inhomogeneous precursor surface coverage Friedli and Utke [18] have developed a GIS simulator program which predicts the gas flux distribution from a circular microtube on a substrate. This GIS simulator uses a three-dimensional Monte Carlo method for rarefied gas flow to model the number of precursor molecules impinging on a sample position. These simulations of the local precursor impinging rates showed also a good correlation to the deposited volume of FEBID-synthesized materials (using the same impinging geometry). However, an experimental confirmation based exclusively on the flux of impinging gas molecules (and not based on the deposition volume) would be beneficial since the validation via the deposited volume may be compromised by (1) the electron-interaction cross section during the deposition step and (2) by surface diffusion of the precursor.

In this work we present an experimental approach to map and to quantify the flux of precursor molecules impinging on different positions of a virtual sample plane. As substitute for a metallorganic precursor we used the noble gas argon, which is known for its extremely low sticking coefficient on inorganic surfaces [19]. Instead of a real sample we use a $0.15 \mu \mathrm{m}^{2}$ collection tube that is (a)

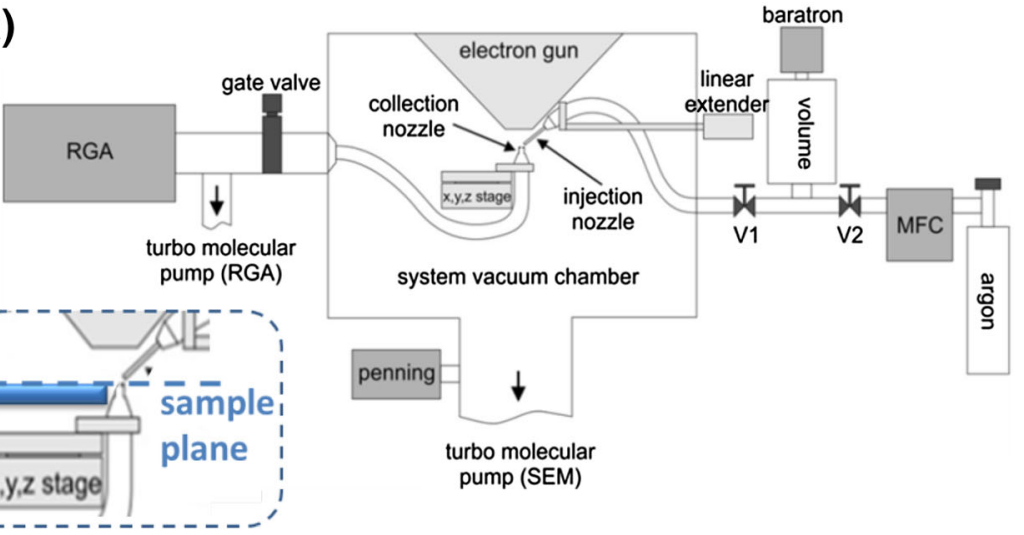

(b)

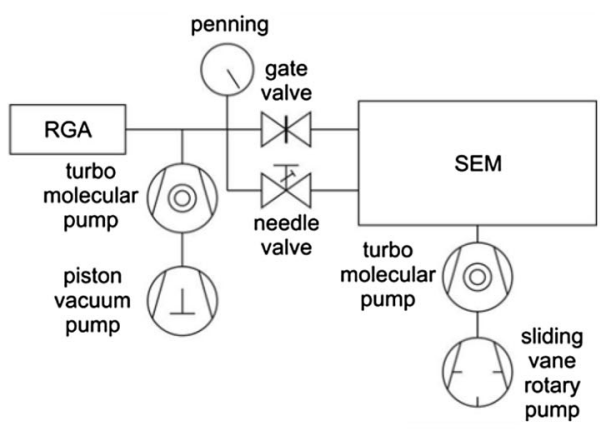

Fig. 1 Schematic illustration of experimental setup a experimental setup for the gas flux distribution measurement. The GIS nozzle is mounted on a linear extender and connected to the gas supply line outside of the SEM chamber. The collection nozzle of the sniffer probe is vertically mounted on the movable xyz stage. The RGA is directly connected to a collection nozzle acting as sniffer probe b RGA attached to the SEM chamber. The two separate pumping systems allow independent operation of the SEM and the RGA 
movable in the exact plane, where usually the sample would be located (Fig. 1). The argon gas was injected through a microtube nozzle of a GIS used also in previous works $[20,21]$. The local gas distribution is measured with a sniffer probe attached to a movable $x-y$ stage, allowing a full mapping of a virtual sample plane. The sniffer probe collecting the argon gas was directly connected to the residual gas analyzer (RGA). Calibration of the quadrupole mass spectrometer was necessary for the quantification of the real argon flow. The distribution of the impinging gas molecules-without interference by surface diffusion or reaction yield -is presented. Furthermore, we performed a comparison between experimental results and simulations executed with the GIS software developed by Friedli and Utke $[18,22]$.

\section{Experimental}

The gas flux measurements were performed in an LEO 1530 VP scanning electron microscope with a 3-stage differentially pumped electronoptical column operating at an acceleration voltage between 0.5 and $30 \mathrm{kV}$ and a beam current between $20 \mathrm{pA}$ and $5 \mathrm{nA}$. The vacuum system of the SEM is based on a Varian Navigator 301 turbomolecular pump with a $\mathrm{N}_{2}$ pumping rate of $280 \mathrm{1} / \mathrm{s}$ in the pressure range between $10^{-6}$ and $10^{-3}$ mbar and an oil-free BOC Edwards XDS10 scroll pump. Samples and the sniffer probe were mounted on a mechanical stage with a nominal positioning accuracy of $1 \mu \mathrm{m}$. The sample stage movement could also be computer-controlled so that also automatic scripts could be run. The precursor was injected via a custom-built proprietary GIS.

The GIS used in this study consists of a reservoir part outside of the vacuum chamber and a nozzle part inside of the vacuum chamber; the parts were connected by a vacuum feed-through. The main components of the outside part are a MKS stainless steel mass flow controller $(5 \mathrm{sccm}$, calibrated for Ar), a stainless steel precursor reservoir with $460 \mathrm{~cm}^{3}$ reference volume, a MKS capacitive pressure gauge (Baratron), a Swagelok dosing valve, a Swagelok shut off cock, and Swagelok connectors for a $1 / 4^{\prime \prime}$ tube system. The inside part of the GIS consists of a stainless steel nozzle with an outside diameter of $600 \mu \mathrm{m}$ and an inner diameter of $350 \mu \mathrm{m}$, an adaptable nozzle holder mounted at a tilt angle of $35^{\circ}$, an $x-y-z$ movable positioning setup for the nozzle and a vacuum-proven, chemically inert Teflon tube connecting the nozzle to the outside part of the GIS via a commercial vacuum feed-through. In this study instead of injecting e.g. a metal organic precursor into the chamber we used argon 9.5 (Linde, grade 5.0). The position of the GIS nozzle under the electron beam was adjusted manually and controlled in the SEM image.
For detection of injected gas a residual gas analyzer (RGA) SRS 300 from Stanford Research Systems (SRS) was attached to the SEM chamber (Fig. 1). The RGA is equipped with a faraday cup detector as well as with an electron multiplier detector and the quadrupole mass filter allows for separation of the detected mass up to $300 \mathrm{amu}$. The RGA is evacuated to a base pressure below $2 \mathrm{E}-7 \mathrm{mbar}$ by its own, independent Varian turbo-molecular pump station with a pumping rate of $60 \mathrm{l} / \mathrm{s}$. For the gas distribution measurement the RGA port was connected to a sniffer probe with a Teflon tube. The sniffer tube itself was mounted on a stage position where usually the sample is mounted. Hence, the sniffer probe could move in the virtual sample plane. The RGA spectra were recorded automated by a LabView program and evaluated with software from Stanford Research Systems. The RGA could be operated in analog scan mode (full mass spectra recorded) or in faster table scan mode (only selected masses recorded).

For gas flux distribution measurement the gas was collected on numerous local positions in the virtual sample plane using a sniffer probe with its collection nozzle mounted on the specimen stage perpendicular to the substrate plane; i.e. the aperture of the probe was exactly in the sample plane. The sniffer probe consisted of a tube with an inner diameter of $1 \mathrm{~mm}$ capped by a $300 \mu \mathrm{m}$ thin aperture with an orifice (diameter $430 \mu \mathrm{m}$ ) on top of the tube. This smaller orifice reduces the probability of collected precursor to reflow out from the sniffer probe back into the SEM chamber.

A simulation of the gas distribution was performed with a freeware GIS simulator program by Friedli and Utke [22]. The simulator is based on the three-dimensional test-particle Monte Carlo method for rarefied gas flow exiting a circular microtube and predicts the gas flux distribution impinging on a substrate.

\section{Results}

In this study, we experimentally measure the gas-impinging rates on the sample surface. The local gradient of the gas-impinging rate is an important parameter for FEBID processes. For this empirical study, a defined flux of argon was injected into a SEM's vacuum chamber, and the argon impinging on the virtual sample plane was collected by a movable sniffer probe. The local amount of argon was measured by the mass spectrometer of an RGA on up to 1,400 virtual sample positions. The inert monoatomic noble gas argon with $39.95 \mathrm{amu}$ results in a peak at the $\mathrm{m} / \mathrm{z}$ value of $40 \mathrm{amu}$ in the mass spectrum. As chemically inert and non-polar, noble gas argon has a small sticking coefficient on most surfaces at room temperature. Due to the 
low residence time of argon, the surface diffusion becomes a negligible factor in the following experiments. Due to its low sticking, coefficient can be quickly removed again from the processing chamber by pumping, which makes argon suitable for a time-resolved study of the flow behavior.

Argon acts as indicator species; so, any systematic errors due to residual argon in the background pressure had to be excluded first. With a mass spectrum of the chamber gas at background pressure, we first confirmed the absence of argon in the residual gas. The graph in Fig. 2 displays the RGA spectrum of the residual gas in the SEM chamber at the base pressure of $2 \times 10^{-6}$ mbar. The mass spectrum shows no detectable peak at the $\mathrm{m} / \mathrm{z}=40$ but reveals

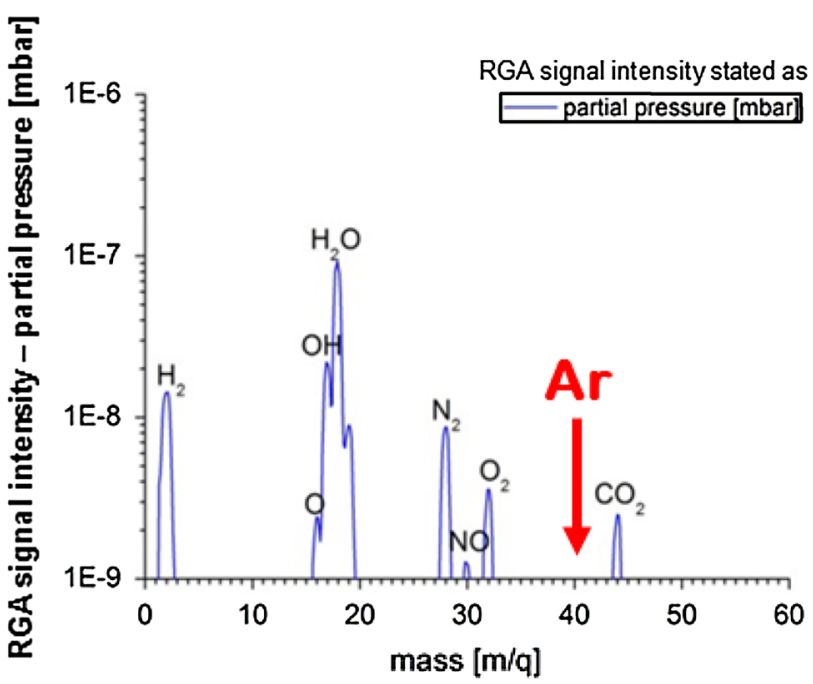

Fig. 2 RGA spectrum of the SEM chamber at base pressure $\left(2 \times 10^{-6}\right.$ mbar $)$ measured through the collection nozzle system. The argon signal at 40 amu was below the detection limit of the RGA

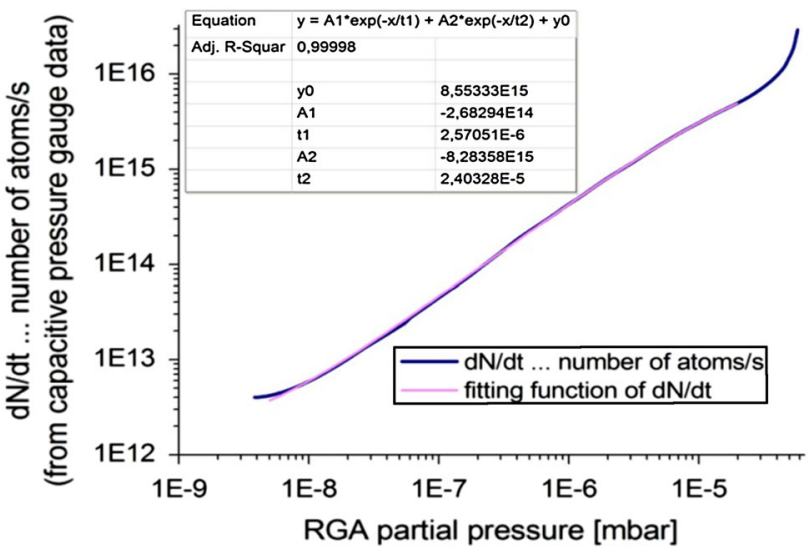

Fig. 3 Calibration curve for the RGA measuring argon atoms. A good fit can be achieved in the range of $5 \times 10^{-9}$ mbar to $2 \times 10^{-5}$ mbar partial pressure with a sum of two exponential functions as displayed in the graph strong peaks at $m / z=18$ and $m / z=17$, indicating a high partial pressure of water. As the used SEM is not equipped with a load-lock system, the residual moisture is the main component of the residual gas. The ratio between $\mathrm{N}_{2}$ and $\mathrm{O}_{2}$ in the SEM chamber is identical to the ratio in air. As argon is absent in the residual gas, this species proved to be suitable as probe for measuring the precursor distribution. The other components in the residual gas provide a stable background which will not interfere with the argon measurements (Fig. 2).

The RGA enables to locally identify the exact composition of the gas collected from the SEM via the actual position of the sniffer probe. However, signal losses by incomplete collection of the gas by the sniffer probe and by incomplete ionization in the RGA do not allow to directly use the signal for quantifying the flow rate. In order to enable the quantification of the absolute rates of molecules impinging on the sample position, the RGA was calibrated (Fig. 3) so that the exact number of argon atoms per second being detected with the collection nozzle (at each measured position of the sample plane) could be measured. This calibration of the flow was performed by correlation of the exactly known amount of gas molecules with the correspondingly collected RGA signal. For calibration, the argon flow passing the injection nozzle was exactly determined by measuring the pressure loss in the reference volume of $460 \mathrm{~cm}^{3}$ using a capacitive pressure gauge (Baratron) (Fig. 3). For the calibration procedure, the injection nozzle was directly linked to the sniffer inlet of the RGA. This setup ensured that the entire amount of argon exiting the GIS was quantitatively injected in the RGA.

Before filling the $460 \mathrm{~cm}^{3}$ reference volume, it was evacuated through the sniffer probe until a base pressure lower than $1 \mathrm{E}-3$ mbar was reached. Afterward the volume was filled with argon gas to an exact pressure of 1 mbar while the valve $V 1$ to the SEM chamber with the RGA was still closed preventing any outflow. At stationary conditions for an almost ideal gas such as argon, the number of atoms in the constant volume can be directly calculated from the ideal gas law. When opening the valve $V 1$ to the system vacuum chamber, the reference volume of $460 \mathrm{~cm}^{3}$ was evacuated through the injection nozzle, which had a lossless connection to the collection nozzle of the RGA. The used flow rates and the corresponding Knudsen-numbers are listed in Table 1.

Simultaneously to the RGA measurement, the absolute flow of argon atoms per second was calculated from the rate of pressure decrease using Eq. 1. The pressure decrease per time period is proportional to the number of molecules effusing from the reference volume through the injection nozzle into RGA. The flow rate $R$ of the effusing molecules is described by $\mathrm{d} N / \mathrm{d} t$ in molecules per second, 
Table 1 The calculated flow rates in molecules per second, the mean free path, and the Knudsen number are listed for the reference gas Ar

\begin{tabular}{llllll}
\hline Flow rate $(\mathrm{sccm})$ & Flow rate $($ molecules/s) & Molecule & Molecule diameter $\sigma(\mathrm{m})$ & Mean free path $\sigma(\mathrm{m})$ & Knudsen number Kn \\
\hline 0.0884 & $3.98 \times 10^{+16}$ & Ar & $3.76 \times 10^{-10}$ & $3.79 \times 10^{04}$ & 1.08 \\
\hline
\end{tabular}

These flow rates were used during the gas flux distribution experiments

in Eq. 1 ( $p$ stands for the current pressure in the reference volume $V, k_{\mathrm{B}}$ is the Boltzmann constant, and $T$ stands for the temperature).

$R=\frac{\mathrm{dN}}{\mathrm{d} t}=\frac{\mathrm{dp}}{\mathrm{d} t} \frac{V}{k_{\mathrm{B}} T}$

The evaluated flow rate at 1.0 mbar argon supply line pressure was $3.98 \times 10^{+16}$ molecules per second corresponding to a flow of $0.0884 \mathrm{sccm}$. Initially, the full calibration curve for the argon flux as a function of the RGA signal has been measured. The derivative function over the corresponding measured RGA signal is displayed in Fig. 3 and reveals an approximately linear correlation between the RGA signal and the gas flow rate of molecules in the range of $2 \times 10^{-8}-1 \times 10^{-6}$ mbar. The signal was fitted by an additive function of two exponential functions showing an excellent correlation with experimental data in the range of $5 \times 10^{-9}-2 \times 10^{-5}$ mbar partial pressure. This fitting function was used for the quantification of the gas flux as measured by the RGA.

In the next step, the lateral distribution of argon in the virtual sample plane was determined. The direct adsorption from the gas phase is an important mechanism for the replenishment of precursor during the deposition process. Small variations in the gas-impinging rate due to a different lateral position may significantly influence the deposition rate. The sniffer probe, which was mounted in the sample plane of a movable stage, collected the argon at different positions under the fixed injection nozzle. So the RGA could quantify the collected argon in dependence on the position of the stage and hence with regard to the nozzle distance. This setup simulates the identical precursor impinging rate as would be experienced on the sample surface during FEBID. With the calibration curve in Fig. 3, the number of locally impinging gas molecules can be directly determined from the number of gas molecules detected by the RGA.

The sniffer probe mounted on the stage was moved around position in close vicinity of the nozzle exit in semicircles with increasing radius in the range of $0.0-2.25 \mathrm{~mm}$. Following this programmed path, the stage moved along different positions in the xy plane of the virtual sample. The relative position of the extractor nozzle with regard to the injection nozzle as well as the horizontal orientation of the injection nozzle was counterchecked by the SEM image. Whenever the stage was moved to a new

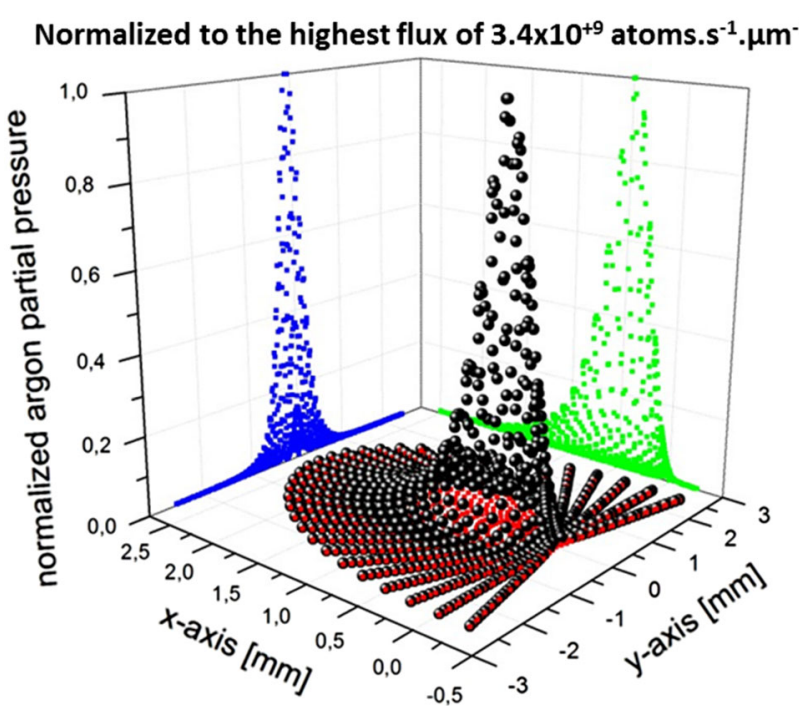

Fig. 4 The measured gas flux distribution is displayed in the figure. Each black dot corresponds to one of the more than 1,400 measured positions. The Ar-intensities were normalized to the maximum flux of $3.4 \times 10^{+9}$ atoms s${ }^{-1} \mu \mathrm{m}^{-2}$ recorded. Also a projection of the measured fluxes in the $x$-plan and in the $y$-plane is given

position, a delay time of $2 \mathrm{~s}$ was permitted before each measurement to allow for stabilization of the stage position and of the argon flux conditions. Subsequently, four measurements were performed with the RGA, and the average value was recorded. The measured distribution of the gas flux of argon corresponds to the position-dependent precursor impinging rates on a virtual sample during a FEBID process.

The gas distribution on the virtual sample was measured for a total argon flow of $3.98 \times 10^{+16}$ atoms s${ }^{-1}$ passing through the injection nozzle. For $350 \mu \mathrm{m}$ inner diameter of the cylindrical nozzle tube, this corresponds to an average flux of $1.034 \times 10^{+11}$ atoms s${ }^{-1} \mu \mathrm{m}^{-2}$. The GIS nozzle was directly touching the virtual sample plane (height $=0 \mu \mathrm{m}$ ) and had a tilt angle of a $35^{\circ}$ with regard to the virtual sample plane. The result of gas flux distribution is shown in Fig. 4 where each dot represents the impinging gas flux on one measured position; the gas flux impinging on the sample surface is normalized to the highest flux measured by the RGA, which was $3.4 \times 10^{+9}$ atoms $\mathrm{s}^{-1} \mu \mathrm{m}^{-2}$ at the impingement center spot. The image shows a smooth surface curve of the gas distribution. Location-dependent quantification of the argon flux 
showed that a slight change of the extractor nozzle position immediately resulted in a significant change of the detected RGA signal. With increasing distance from the impingement center spot, the gas flux quickly decays to $50 \%$ within $\sim 300 \mu \mathrm{m}$. This underlines the importance of choosing the proper impingement geometry for consistent FEBID results. This observation also plausibly illustrates why data from different authors using different GIS hardware and undisclosed sample-nozzle geometries can hardly be compared in literature. Our results prove the importance of a better documentation of these experimental parameters in future publications of the FEBID community.

It has to be noted that, in this gas distribution study, the described RGA quantification was selective for the reference species argon and had no cross-sensitivities to other residual gas species. For real FEBID with a precursor gas, also parasitic location-independent adsorption from the residual gas is an additional component, which unintentionally adds to the deliberate precursor adsorption originating from the nozzle. This adsorption of residual gas originating from the SEM background pressure may also influence the precursor coverage and also lead to parasitic deposition of contaminants. Taking into account a base pressure of $1.2 \times 10^{-5}$ mbar in the SEM chamber (as experienced in this experiment), this contribution of the residual gas leads to an additional, location-independent impinging rate of only $1.32 \times 10^{+7} \mathrm{~mol} \mathrm{~s}^{-1} \mu \mathrm{m}^{-2}$. In the central impinging area featuring the highest impinging rate, the contribution of the residual gas is $<0.4 \%$ of the nozzle-borne impinging rate, while in peripheral areas the contribution from the residual gas will become dominant.

The presented data prove the importance to know and to control the gas flux rates, and researchers may wish to retrieve this information for their own GIS geometry. As our experimental approach would require a complex expansion of the SEM to determine the gas flux distribution, as alternative also simulations of physical processes are highly regarded, for example, a gas flux simulation model has already been developed by Friedli et al. [23] and was indirectly validated by the local distribution of the deposited volumes of FEBID cobalt structures. However, it has to be considered that the deposited volume does not exclusively reflect the flux of impinging molecules-which is the output of the simulation-but at the same time, the material volume is simultaneously influenced by surface diffusion and the electron-dissociation cross section. Consequently, in a final step, we compare our experimental data-which exclusively represents the flux of impinging molecules-with the simulation results of the gas-impinging rates. The gas flux simulation model by Friedli and Utke [22] was exactly adapted to our GIS geometry and used to calculate the gas distribution based on molecule collisions. Both our experiment and the GIS simulation provide comparable data: Both only consider the gas molecules impinging on the sample and do neither involve contributions by surface diffusion nor by the electron-dissociation yield (which influences the deposition reaction). The measured gas flux distribution results (Fig. 5a) and the simulated gas flux distribution (Fig. 5b) are displayed in an iso-contour line graph. Figure 5a represents the same data set as already displayed in Fig. 4.

The simulation result in Fig. 5b states the impinging gas flow for an incrementally small collection area, while the real measurement uses a sniffer probe with a collection aperture of $430 \mu \mathrm{m}$ diameter. Due to the convolution with the finite nozzle geometry in the experiment, the measured flux values average over the collection area and consequently cannot be directly compared with the simulated gas flux distribution. Only the convolution of the simulated gas

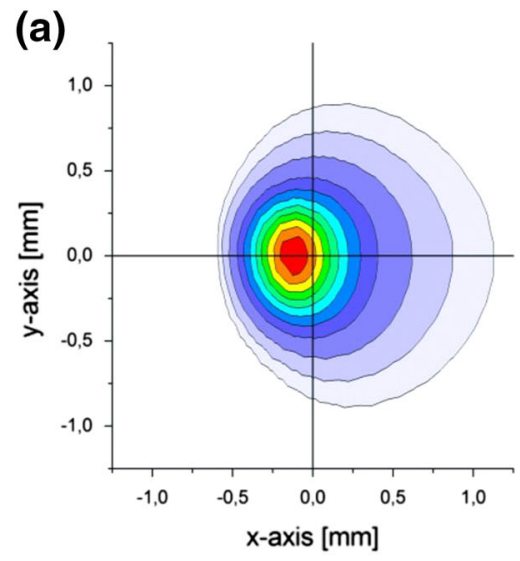

(b)

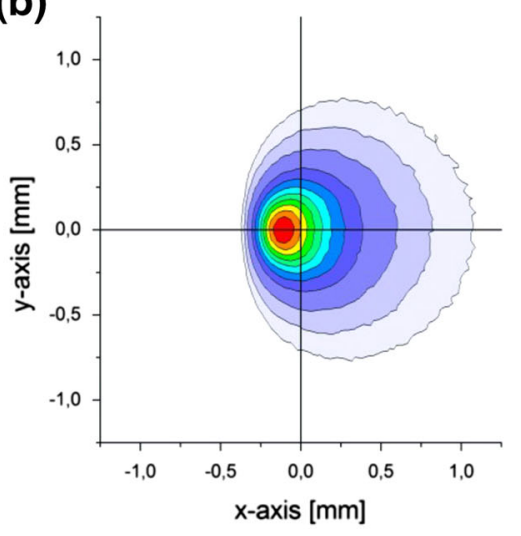

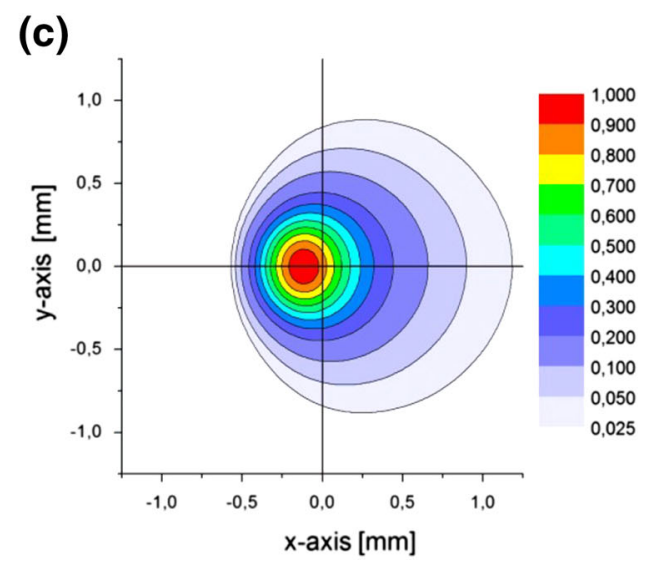

Fig. 5 Normalized iso-contour lines of the gas flux distribution are displayed in the graph. a The evaluated gas flux distribution measured with argon gas and the collection nozzle with the $430 \mu \mathrm{m}$ sized aperture is shown. b The simulated gas flux distribution for the same nozzle and flux parameters as used for the measurement in (a) is denoted. $\mathbf{c}$ The result on the convolution of the simulated gas flux distribution and a circular low pass filter (representing the finite dimension of the collection nozzle) is illustrated 


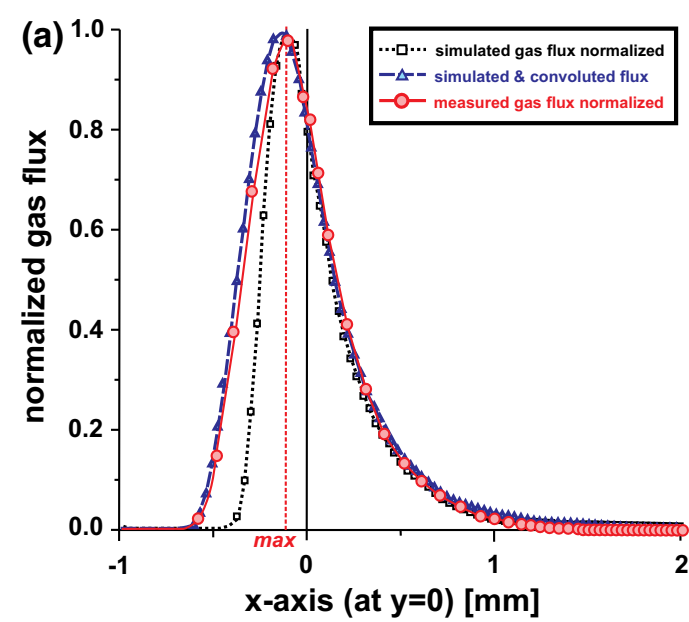

Fig. 6 Cross sections of the measured gas flux distribution (red), the simulated distribution (black), and the convoluted simulated distribution with a circular low pass filter are displayed in the graph along

flux with a circular low pass filter (simulating the circular geometry of the collection aperture with a $430 \mu \mathrm{m}$ diameter) facilitates a meaningful comparison. With a selfwritten MatLab program (see supplement), such a convolution calculation was performed using a Fast Fourier Transformation (FFT) procedure to simulate the conditions of the real physical collection setup. The resulting distribution after the FFT operation is shown in Fig. 5c. In contrast to the mere simulation result (Fig. 5b), this convolution-corrected result (Fig. 5c) matches the experimentally measured gas distribution (Fig. 5a) very well.

For better comparison between the experimental data (Fig. 5a) and the convoluted simulation data (Fig. 5c), a 2D representation of cross sections along the $x$-axis $(y=0)$ and parallel to the $y$-axis ( $x$ at maximum impingement position) is displayed in Fig. 6. Along the $x$-axis, the deviation between measurement and convoluted simulation is $<2 \%$, while in the $y$-direction the differences are $<5 \%$. The experimentally measured signal appears to be slightly broader than predicted by the simulation. A potential reason may be the surface roughness and microscopic deformations and non-idealities of real injection tubes in contrast to the ideally smooth perfect cylinder tubes assumed in the simulation. Overall, the experimentally observed and the theoretically modeled distribution of precursor impinging rates showed a highly satisfactory correlation. Our experimental results fully validate the gas flux simulation model of Friedli et al. [22]. Researchers may model their real precursor flux distribution for their GIS geometry and-based on our experimental evidencemay determine the local precursor flux rates of their experiments a posteriori to make FEBID results comparable within the scientific community.

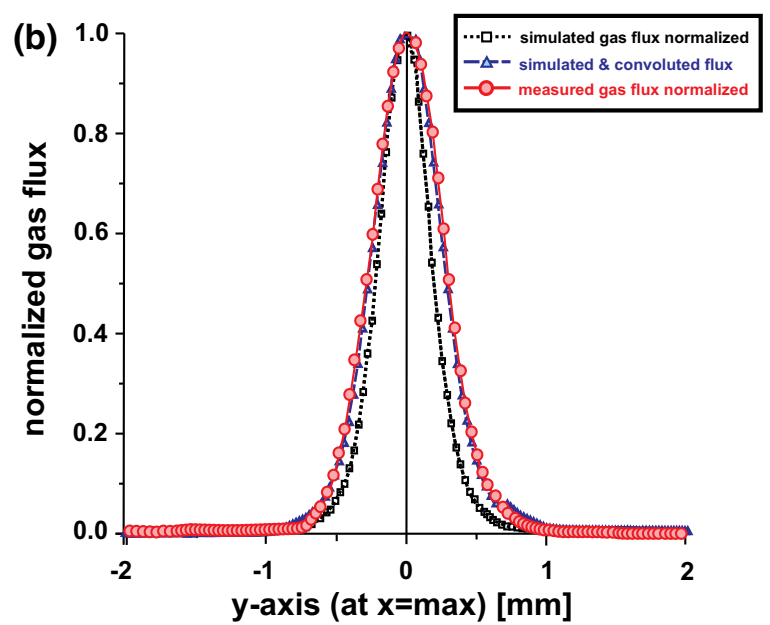

the $\mathbf{a} x$-axis (at $y=0$ ) and $\mathbf{b}$ parallel to the $y$-axis ( $x$ at maximum impingement position)

\section{Conclusion}

In this work, the issue of reproducible precursors supply in a SEM chamber for FEBID applications was experimentally addressed. The experimental setup consisted of a GIS injector nozzle and a collection aperture directly connected to an RGA for gas quantification. Using the inert gas argon as indicator species, the RGA was first calibrated to yield absolute flux rates in atoms $\mathrm{s}^{-1} \mu \mathrm{m}^{-2}$. Subsequently, the distribution of gas atoms impinging on the surface was measured. The gas flux distribution on the surface indicated a strong dependency of the gas impingement rate on the position of the collection aperture. For FEBID, this result also implies a strong gradient of the deposition rate in dependence on the nozzle position. The precise examination of the gas flux-dependent gas impingement rates was accessible due to the precise quantification of the gas flux distribution proven by our experimental approach. The empirical data were confirmed by simulation results gained with a GIS simulator software. The gained know-how on the precursor supply mechanisms to the surface provided a valuable bias for the further interpretation of the deposition experiments. Indeed, the precursor supply contribution from surface diffusion could be successfully distinguished. With the measurement of the deposited volume, also surface diffusion of the precursor and precursor consumption by the electron initiated decomposition are additional factors influencing the resulting deposition geometry.

The results of our work underline the importance of the knowledge of the precise gas flux distribution on top of the surface. For a high reproducibility of all deposition experiments, it is recommended to position the nozzle exit 
in an identical position with regard to the deposition area and to restrict the deposition zone to an area granting a quasi-homogeneous precursor supply. For a straight-cut tube-shaped nozzle $(600 \mu \mathrm{m}$ outer diameter, $350 \mu \mathrm{m}$ inner diameter, $35^{\circ}$ incidence angle, and $0 \mu \mathrm{m}$ up to $600 \mu \mathrm{m}$ vertical height from lowest point over sample), all structures deposited within an area of $50 \mu \mathrm{m}$ diameter have experienced an almost homogeneous precursor supply. The diameter of this recommended sweet spot is in the same range as the vertical distance between the sample plane and the lowest point of the nozzle opening, and its area accounts only for little more than $2 \%$ of the opening area of the injection nozzle.

We encourage all researchers in the field of FEBID to include a close description of the nozzle geometry as this is a precondition for readers to understand the process regime during deposition. As minimum parameter set for tubeshaped nozzles, we suggest to include at least the 4 most important parameters: (1) the tube inner diameter, (2) the tube outer diameter, (3) the tube incidence angle, and (4) the vertical height of the lowest tube point above the sample plane (or alternatively the distance from the center of the tube exit to the surface in direction of the nozzles length axis). Additional description should be provided for non-straight-cut nozzles, or nozzles with side-wall access holes, or short large-bore nozzles where length is not one magnitude larger than the inner diameter. Furthermore, the flow regime and the position of the deposition area with regard to the center of the tube exit should be stated to allow for assessment of the surface conditions. Describing these parameters will not only provide researchers a better understanding of own experiments but will be a step toward the long-desired comparability of experimental results of the FEBID community.

Acknowledgments We acknowledge the Austrian Science Fund (FWF) for financial support under project P24093. We thank V. Friedli and I. Utke (EMPA, Switzerland) for providing the GIS simulator software for modeling of the gas distribution. We thank Carl Zeiss NTS (Oberkochen, Germany) for their support.

Open Access This article is distributed under the terms of the Creative Commons Attribution License which permits any use, distribution, and reproduction in any medium, provided the original author(s) and the source are credited.

\section{References}

1. I. Utke, P. Hoffmann, J. Melngailis, J. Vac. Sci. Technol. B 26, 1197 (2008)

2. J.D. Fowlkes, D.A. Smith, M.G. Lassiter, P.D. Rack, Microsc. Microanal. 15, 318 (2009)

3. C. Schoessler, H.W.P. Koops, J. Vac. Sci. Technol. B 16, 862 (1998)

4. P. Hoffmann, I. Utke, F. Cicoira, Proc. SPIE 5023, 4 (2003)

5. I. Utke, F. Cicoira, G. Jaenchen, P. Hoffmann, L. Scandella, B. Dwir, E. Kapon, D. Laub, Ph. Buffat, N. Xanthopoulos, H.J.Mathieu, Materials research society symposium-proceedings 706, 307-312 (2002)

6. S. Engmann, M. Stano, P. Papp, M.J. Brunger, Š. Matejčík, O. Ingólfsson, J. Chem. Phys. 138, 044305 (2013)

7. S. Engmann, M. Stano, T. Matejík, O. Ingólfsson, J. Phy, Conf. Ser. 388, 052019 (2012)

8. H.D.W. Vinzenz Friedli, I. Utke, "Gas injection systems" in "nanofabrication using focused ion and electron beams principles and applications" Eds. I. Utke, S. Moshkalev, and P. Russell, vol. Gas injection systems for FEB and FIB processing: theory and experiment, (2012)

9. I. Utke, V. Friedli, M. Purrucker, J. Michler, J. Vac. Sci. Technol. B 25, 2219 (2007)

10. D.A. Smith, J.D. Fowlkes, P.D. Rack, Small 4, 1382 (2008)

11. R. Winkler, J. Fowlkes, A. Szkudlarek, I. Utke, P.D. Rack, H. Plank, ACS Appl. Mater. Interfaces 6, 2987 (2014)

12. A.V. Riazanova, B.N. Costanzi, A. Aristov, Y.G.M. Rikers, V. Ström, J.J.L. Mulders, et al., Nanotechnology. 25, DOI: 10.1088/ 0957-4484/25/15/155301 (2014)

13. H.D. Wanzenboeck, M. Fischer, J. Gottsbachner, S. Mueller, W. Brezna, M. Schramboeck, E. Bertagnolli, ECS Trans. 5th Int. Symp. Phys. Chem. SiO2 and $\mathrm{Si}-\mathrm{SiO} 2$ Interface 1, 69 (2005)

14. P.C. Hoyle, M. Ogasawara, J.R.A. Cleaver, H. Ahmed, Appl. Phys. Let. 62, 3043 (1993)

15. J.D. Fowlkes, S.J. Randolph, P.D. Rack, J. Vac. Sci. Technol. B 23, 2825 (2005)

16. K. Rykaczewski, W.B. White, A.G. Fedorov, J. Appl. Phys. 101, 054307-054311 (2007)

17. M.S. Gabureac, L. Bernau, I. Utke, J. Nanosci. Nanotechnol. 11, 7982 (2011)

18. V. Friedli, I. Utke, J. Phys. D Appl. Phys. 42, 125305 (2009)

19. D.J. Harra, J. Vac. Sci. Technol. 13, 471 (1976)

20. G. Hochleitner, H.D. Wanzenboeck, E. Bertagnolli, J. Vac. Sci. Technol. B 26, 939 (2008)

21. M. Gavagnin, H.D. Wanzenboeck, D. Belić, E. Bertagnolli, ACS Nano 7, 777 (2013)

22. I. Utke. (2013). GIS simulator software. http://www.empa.ch/ plugin/template/empa/1091/*/- $/ 1=1$

23. I. Utke, V. Friedli, S. Amorosi, J. Michler, P. Hoffmann, Microelectron. Eng. 83, 1499 (2006) 